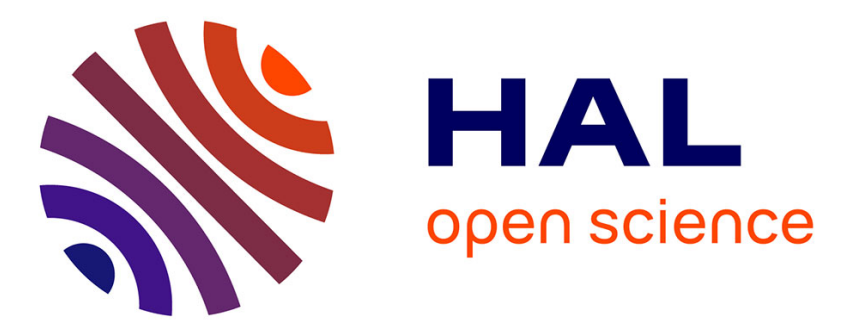

\title{
Le PKK, parti des femmes. La constitution du sujet politique féminin entre virilisme combattant et féminisme martial
}

\author{
Caroline Guibet Lafaye
}

\section{- To cite this version:}

Caroline Guibet Lafaye. Le PKK, parti des femmes. La constitution du sujet politique féminin entre virilisme combattant et féminisme martial. Pôle Sud - Revue de science politique de l'Europe méridionale, 2022. hal-03558775v2

\section{HAL Id: hal-03558775 \\ https://hal.science/hal-03558775v2}

Submitted on 30 Jan 2023

HAL is a multi-disciplinary open access archive for the deposit and dissemination of scientific research documents, whether they are published or not. The documents may come from teaching and research institutions in France or abroad, or from public or private research centers.
L'archive ouverte pluridisciplinaire HAL, est destinée au dépôt et à la diffusion de documents scientifiques de niveau recherche, publiés ou non, émanant des établissements d'enseignement et de recherche français ou étrangers, des laboratoires publics ou privés. 


\title{
LE PKK, PARTI DES FEMMES.
}

\section{LA CONSTITUTION DU SUJET POLITIQUE FEMININ ENTRE VIRILISME COMBATTANT ET FEMINISME MARTIAL ${ }^{1}$}

\author{
Caroline Guibet Lafaye \\ DR \\ CNRS \\ caroline.guibetlafaye@univ-tlse2.fr
}

\begin{abstract}
Résumé : Certaines organisations clandestines ainsi que les medias occidentaux font très volontiers la promotion de la part prise par les femmes combattantes dans des guérillas comme celle du Partiya Karkerên Kurdistan (PKK). Au-delà des développements idéologiques de la théorie de la Femme libre, le statut des femmes a fait l'objet d'une conquête féminine menée avec peine au sein du parti. Afin d'en apprécier les évolutions, nous nous sommes appuyées sur une enquête de sociologie qualitative menée auprès de 28 combattantes du PKK. Après un bref retour sur la phase de l'«armification » des femmes, nous mettons en évidence les formes de discrimination de genre persistantes au sein du parti depuis les années 1990 et soulignons le rôle de l'«armification» des femmes dans l'institution de soi comme sujet politique. Ces éléments nous conduisent à reconsidérer la thèse du féminisme martial, développée dans la littérature académique et pensée idéologiquement comme un «outil et une condition de l'émancipation des femmes », pour la nuancer à partir du regard que portent les femmes sur leur rapport à la violence politique.
\end{abstract}

Mots-clefs : PKK, théorie de la Femme libre, Kurdes, émancipation, féminisme martial.

Abstract: Some clandestine organisations as well as the Western media willingly promote the part played by women fighters in guerrilla movements such as the Partiya Karkerên Kurdistan (PKK). Beyond the ideological developments of the "Free Woman" theory, the status of women was the object of a difficult women's conquest carried out within the party. In order to appreciate its evolution, we have relied on a qualitative sociological survey conducted among 28 female PKK fighters. After a brief look back at the phase of the 'armification' of women, we will highlight the forms of gender discrimination that have persisted within the party since the 1990s and emphasise the role of the 'armification' of women in the institution of the self as political subject. These elements will lead us to reconsider the thesis of martial feminism, developed in the academic literature and ideologically thought of as a "tool and a condition of women's emancipation", in order to nuance it from the women's relationship to political violence.

Key words: PKK, theory of the Free Woman, Kurds, emancipation, martial feminism.

${ }^{1}$ Article paru dans Pôle Sud. Revue de science politique de l'Europe méridionale, Montpellier, vol. 56, nº 1, 2022, p. 97-116. 
« Il n'est pas question chez nous d'un problème de droits des femmes, mais de la

libération de "la" femme, et de la question de savoir comment elle pourra se réintroduire dans les rangs de l'humanité »². » (« Frauen in Kurdistan », 1988)

\section{Introduction}

Au sein du Parti des travailleurs du Kurdistan (Partiya Karkerên Kurdistan - PKK), une « armée des femmes » a été formée en 1993 consistant en unités militaires non-mixtes. A suivi, dans le domaine politique, la constitution du parti des femmes ou Parti du travail des femmes du Kurdistan (Partiya Finên Karkerên Kurdistan - PJKK) en 1999. Il a été porté par des militantes du PKK et créé pour mettre en œuvre l'idéologie de la libération des femmes. Le PJKK a été rebaptisé Parti des femmes libres (Partiya finên Azad - PJA) lors du congrès de 2000 au cours duquel la thèse de la "République démocratique » est adoptée et définitivement accepté l'abandon de la lutte pour un État kurde indépendant. Dans ce cadre, l'expression de «femmes libres» se voit privilégiée du fait de ses connotations universelles (KJK, 2013). De même, le "Contrat social des femmes", qui rassemble les débats menés par le parti des femmes, est considéré comme le nouvel objectif social du mouvement (Serok, 2001). Le Parti des femmes libres du Kurdistan (Partiya Azadîya Finên Kurdistan - PAJK), fondé en 2004, se transforme en 2005 dans la confédération de l'Association suprême des femmes (Koma finên Bilind - KJB), censée inclure toutes les organisations de femmes dans la sphère d'influence du mouvement. La KJB est remplacée, lors de sa septième convention extraordinaire en 2014, par les Sociétés de femmes du Kurdistan (Komalên Finên Kurdistan - KJK).

Quoique l'auto-organisation procède en 1993 d'une initiative féminine, l'implication active des femmes dans le mouvement a, à l'origine, été fortement liée à la direction du parti, à son leader Abdullah Öcalan. Dès la fondation du PKK en 1978, celui-ci a procédé à un travail de redéfinition du contour des identités ethniques et de genre existantes. Par ses réflexions théoriques, Öcalan a contribué à une redéfinition du rôle des femmes dans la famille et au sein du peuple kurde, à la construction du mythe d'un âge d'or néolithique et d'une organisation ancestrale autonome des femmes. L'articulation du masculin et du féminin dans ce processus d'autonomisation a connu des soubresauts puisqu'A. Öcalan dissout le premier «Congrès National des Femmes » en 1992 après l'avoir préliminairement soutenu. De même, lorsque les unités militaires autonomes sont constituées, elles sont directement liées à Öcalan et l'appui de ce dernier est requis pour contourner les schémas hiérarchiques de la politique du parti pour appliquer leurs décisions (Çağlayan, 2020, p. 76). La place incontournable assumée par Öcalan dans cette évolution a eu pour effet de provoquer une crise temporaire entre l'administration du parti et les unités autonomes de femmes après son emprisonnement en 1999 (Çağlayan, 2020, p. 76). En effet, le Conseil présidentiel, secondant Öcalan dans la hiérarchie du parti, s'est opposé aux tentatives féminines de maintenir une autonomie et la revendication d'engagement (Jongerden, 2017, p. 245).

Sur le plan des représentations traditionnelles, la répartition des rôles de genre veut que les hommes aient pour mission de protéger les frontières de la nation, et que les femmes et leurs corps soient les gardiens de ces frontières (King, 2008). Au Moyen-Orient comme en Occident, femmes et hommes sont pris dans des rapports sociaux de sexe et une division sexuelle du travail (Kergoat, 2000). L'évolution de ces représentations, dans le monde kurde, n'est pas simplement le fait des féministes, qui n'ont pas manqué de critiquer cette représentation des rôles de genre dans la région (Yüksel, 2006), ni celui des théories proposées par Öcalan (1992a, 1992b, 1993). Cette mutation doit davantage à l'engagement des femmes

2 ERNK's German publicity organ, « Frauen in Kurdistan », Kurdistan Report, vol. 24, 1988. 
dans la guérilla kurde et à leur médiatisation. La figure de la femme combattante a joué un rôle central dans la transformation du discours nationaliste sur le genre. Toutefois une image très spécifique de la femme kurde combattante a été promue dont il n'est pas certain qu'elle ne soit pas encore véhicule de stéréotypes de genre.

La participation des femmes à la violence politique demeure, aujourd'hui encore, perçue comme une transgression. Les combattantes placées sur le devant de la scène ne sont pas présentées comme des guerrières hyper-masculinisées mais comme des hérö̈nes féminines. L'archétype de cette représentation s'est cristallisé dans la médiatisation de la combattante féminine, belle et héroïque contre les «barbares » misogynes de l'État islamique, véhiculée par les médias occidentaux (Begikhani et al., 2018, p. 18 ; Toivanen et Baser, 2016, p. 306 ; Allison, 2001). Les femmes se dresseraient face à l'oppression patriarcale (moyenâgeuse) de leurs adversaires. L’imagerie proposée par les médias français et britanniques, mais également kurdes $^{3}$, produit une représentation des combattantes les distinguant de leurs homologues masculins $s^{4}$. Lorsqu'elles ne sont pas sur le front, les femmes sont souvent présentées en train de rire, de danser, de jouir de la vie. Ainsi «la guerre est devenue féminine, et les guerriers autrefois hyper-masculinisés ont été investis de caractéristiques typiquement féminines inhérentes (mais stéréotypées) » (Weiss, 2018).

Cependant la fétichisation des combattantes des Brigades d'autodéfense féminines ou Unités de protection des femmes (Yekineyên Parastina fin - YPJ) et des Brigades générales d'autodéfense ou Unités de protection du peuple (Yekîneyên Parastina Gel - YPG) au Kurdistan ainsi que des unités non-mixtes du PKK tend à occulter l'implication, les conséquences et les obstacles rencontrés par l'engagement clandestin des femmes, issues de sociétés patriarcales au Moyen-Orient ${ }^{5}$. Pourtant « les YPJ ne se battent pas seulement contre l'État islamique, elles se battent pour le féminisme et l'égalité des sexes - et elles le font aussi bien avec des idées qu'avec des balles » (Dirik, 2015). Au-delà de la présentation sensationnaliste des combattantes kurdes, il convient à la fois d'interroger la réalité de leur intégration historique au sein de la guérilla, en l'occurrence du PKK, et l'évolution inhérente des stéréotypes de genre dans le parti. Il importe également de comprendre le rôle dont peut être investi, à la fois au sein de celui-ci et pour son image extérieure, la mise au premier plan des femmes combattantes. Ce sont ces questions que nous envisagerons en revenant, dans un premier temps, sur l'évolution des rapports de genre au sein de la guérilla, à partir d'une enquête de terrain réalisée auprès de combattant.e.s (voir Annexe pour les détails de cette dernière). Nous considérerons ensuite la constitution du sujet politique féminin à travers ce qu'il est convenu d'appeler l'armification des femmes, c'est-à-dire sur l'investissement d'une fonction émancipatrice, pour le peuple kurde, de la participation des femmes aux unités militaires de la guérilla. Nous reviendrons enfin sur la réalité du «féminisme martial » ${ }^{6}$ et sa prégnance dans le champ académique occidental. Celui-ci sera compris non pas comme une idéologie sacrificielle (Schalk, 1994) mais comme un rapport à la lutte armée comme outil d'émancipation du peuple opprimé et vecteur de la libération des femmes, pour autant que leur rôle militaire en font des égales des hommes et les distingue fondamentalement des femmes assumant des tâches domestiques.

\footnotetext{
${ }^{3}$ Ainsi «le corps féminin peut encore représenter une bravoure, un héroïsme et un renouveau spécifiquement kurdes, du moins aux yeux d'une grande partie des partisans kurdes » (Begikhani et al., 2018, p. 19).

${ }^{4}$ On parviendrait aux mêmes conclusions à partir de l'imagerie de la démobilisation des FARC en Colombie.

${ }^{5}$ Notons toutefois que cette hypermédiatisation dirige le projeteur sur les YPG plutôt que sur le PKK, les premières ayant collaboré avec la coalition occidentale dans son intervention contre l'État islamique en 2014-2015.

${ }^{6}$ Sur la notion de « féminisme martial », voir Gayer, 2019.
} 


\section{L'armification des femmes}

À partir de la toute fin des années 1980 et en dépit des freins qu’y oppose A. Öcalan, les combattantes tentent de se structurer en unités politique et militaires non-mixtes. Le congrès de l'Union des femmes patriotes du Kurdistan (Yekîtiya finên Welatparêzên Kurdistan - YJWK) a lieu en 1987 en Europe. À cette époque, les combattantes se forment mutuellement, en lisant collectivement des écrits de féministes "comme ceux de Rosa Luxembourg ou Clara Zetkine $»^{7}$ et en échangeant sur les violences spécifiques qu'elles subissent, en plus de la lecture obligatoire des écrits d'A. Öcalan. Des divisions non-mixtes sont créées en 1992 ${ }^{\circ}$. Jusqu'au début des années 1990, aucune femme ne pouvait espérer devenir commandante : on estimait alors que les femmes n'avaient pas encore assez confiance en elles pour se faire respecter (Grojean, 2013, p. 28). Néanmoins se constituent dans les provinces kurdes du sud-est de la Turquie, des «mangas» et des unités non-mixtes dès les années 1990. L'entraînement physique et l'éducation politique ont donné aux femmes une confiance dans leurs capacités personnelles à participer à parité avec les hommes à la lutte de libération nationale ${ }^{9}$. "La croyance que les femmes n'étaient pas capables, ni au niveau émotionnel ni au niveau physique de s'entraîner et de combattre, se trouvait contestée puisque les femmes se prouvaient à elles-mêmes qu'elles étaient aussi engagées et aussi capables que les hommes. Leur force se manifestait à leurs yeux propres et à ceux des autres. » (Peteet, 1991, p. 149)

La non-mixité a permis en outre aux combattantes du PKK d'accéder, à la fin des années 1990, à des postes à responsabilité (Guillemet, 2017, p. 72-73). À partir de la fin des années 1990, certaines deviennent commandantes de régions entières. Les Forces de Défense du Peuple (Hêzên Parastina Gel - HPG), unité armée du PKK, sont composées de plus d'un tiers de femmes. Celles-ci fondent en 2000 l'unité non-mixte des YJA-Star (Yekitiya finen Azad Unités des femmes libres). Ce groupement militaire du PKK relève du PAJK, parti non-mixte au sein du parti. Ce modèle d'organisation est importé par le PKK dans chaque partie du Kurdistan. Plus tard, avec la création des unités féminines, les combattantes ont progressivement pris place au sein des directions des régions militaires en tant que commandantes d'unités. Contrairement à ce qui a pu être le cas dans des corps détenteurs de la violence légitime, l'incorporation des femmes dans les rangs de cette guérilla ne s'est pas réalisée selon un "principe de dissémination des femmes » (Pruvost, 2007), pour des raisons qui tiennent notamment à l'organisation des rapports sociaux de sexe dans la société kurde. En revanche, dans la guérilla, l'entrée dans des corps militarisés intervient comme un vecteur d'émancipation: "Dans le cas des combattantes kurdes, la non-mixité est un outil pour construire un rapport de force triple et participer petit à petit de leur autonomisation des structures patriarcales et des oppressions auxquelles elles font face, en tant que femmes et en tant que Kurdes. » (Guillemet, 2017, p. 75) Il s'agit pour les combattantes de se construire non pas seulement comme actrices militaires mais comme «sujets» politiques. Ces unités nonmixtes contribuent à légitimer la présence des femmes dans les rangs de la guérilla et l'intégration de revendications féministes aux projets politiques de leurs propres partis. «Les camps du PKK comme ceux du Komala, mixtes ou non-mixtes, deviennent des espaces d'expérimentation des idéaux politiques. » (Guillemet, 2017, p. 76) Ces unités s'associent à des

\footnotetext{
7 Extrait d'entretien mené par S. Guillemet, août 2015, Bordeaux.

${ }^{8}$ Sur le premier " Congrès National des Femmes », voir introduction.

9 Il semblerait qu'au début des années 1990 le règlement de l'Académie Mahsum Korkmaz prévoyait que seuls les hommes s'occupaient de l'intendance (cuisine et nettoyage) parce que les femmes, souvent moins instruites, avaient besoin de davantage de formations mais également parce qu'elles avaient déjà assez donné « lors de ces 5000 ans de patriarcat » (Flach, 2007, p. 107).
} 
formes de disciplines, présentées comme structurantes de l'émancipation féminine dans la guérilla (Abu-Lughod, 1998, p. 256). Berbiheyv en témoigne lorsqu'elle décrit l'organisation des bataillons du YPJ, dont elle a été l'une responsable et qui regroupent des adolescentes de 13 à 16 ans $^{10}$. Nous soulignerons néanmoins les réticences masculines face à leur présence (infra 2.).

La constitution du sujet politique passe notamment par le déploiement d'une force physique, de qualités viriles et martiales qui permettent aux femmes d'apparaître comme des égales de l'homme. Cette injonction est caractéristique des attentes auxquelles les femmes ont été confrontées dans les années 1990, c'est-à-dire lors de la deuxième cohorte d'engagement dans le parti ${ }^{11}$. Les souvenirs de Çiçek (C 2), qui a rejoint le PKK en 1992 à 16 ans, soulignent les médiations et occasions à travers lesquelles les combattantes sont conduites à dépasser leur assignation au statut d'inférieures :

« lls nous ont mis sous le commandement de l'unité d'Heval [camarade] Ali et nous sommes allées à l'entraînement [militaire] comme ça. Toutes les autres camarades femmes sont restées au quartier général avec Ferhat; ils disaient: "Celles-ci [les femmes] ne peuvent pas se battre, ce ne sont que des problèmes, elles ne peuvent rien faire, alors les femmes de cette unité doivent faire leurs preuves !' [...] Un biksi [Kalachnikov] a normalement I5 balles; les nôtres avaient aussi 15 balles. Nous allions sur le terrain; il n'y avait pas de munitions; nous devions porter toutes les munitions. Et c'est difficile de tout porter. Heval Rengin était responsable de notre biksi, elle était aussi le commandant adjoint de notre escouade ; elle était un peu plus âgée que nous. Moi et une autre camarade étions ses assistantes. Heval Rengin transportait environ 500 balles avec son biksi, et nous l'aidions. Donc, avec toutes ces cartouches et ces munitions, nous transportions aussi ces 500 balles de biksi. De plus, en termes géographiques, le Kurdistan

${ }^{10}$ Berbiheyv (C 3) est entrée dans la guérilla en 2005. Interrogée sur la place de la femme dans le mouvement, elle souligne : «Il y a eu une évolution positive. Parce que lorsque je me suis engagée, la femme avait déjà reconnu sa force, elle avait déjà surpassé certaines difficultés. Car avant que je ne vienne, les difficultés vécues par les femmes n'étaient pas les mêmes. La période de mon engagement est une période où la femme a reconnu sa propre force, où les femmes se sont mobilisées et sont devenues un système, je n'ai pas eu de moments difficiles en tant que femme. Je n'ai pas vécu les difficultés rencontrées par les premières camarades qui se sont engagées. Au contraire, il y avait déjà un terrain de prêt. J'ai appris à me connaître sur ce terrain-là. J'ai progressé en tant que femme, j'ai immédiatement ressenti cette signification si spéciale de lutter en tant que femme, parce qu'il y a [un] mouvement des femmes prêt et systématisé. C'est pourquoi il y a une évolution dans un sens positif. Il y a eu davantage de progrès. Plus les femmes se sont engagées, plus elles se sont renforcées, à la fois physiquement et qualitativement. Cela donne au peuple plus de force, d'enthousiasme, tu comprends que tu t'engages et progresses davantage en tant que femme, à mesure que le mouvement des femmes progresse. Tu as alors la conviction que ce mouvement et cette société ne peuvent être mis en valeur ni progresser sans les femmes. Plutôt qu'une évolution, c'était un développement positif. »

${ }_{11}$ Nous renvoyons à l'analyse que nous avons proposée des trois générations sociales d'engagement au sein du PKK de la constitution du parti à nos jours (Guibet Lafaye et Tugrul, 2022 ; Guibet Lafaye, 2022a, 2022b). Une enquête réalisée entre mars 2016 et fin 2017, auprès de 28 combattantes et de 36 guérilleros du PKK, nous a permis de mettre en évidence trois vagues d'intégration du parti : la génération d'avant 1990 (C 1), la massification de l'engagement durant les années 1990 (C 2), la génération ayant intégré le parti après l'arrestation d'A. Öcalan (C 3) (Guibet Lafaye et Tugrul, 2022 ; Guibet Lafaye, 2022). L'Annexe présente la liste des répondantes et leurs caractéristiques sociodémographiques. Elle précise également les conditions dans lesquelles l'enquête s'est réalisée. L'étude générationnelle à partir de l'enquête de terrain, dans le cas particulier des femmes du PKK, est confrontée au nombre restreint de celles qui se sont engagées durant les premières années de fondation puis de développement du parti. Nous n'avons rencontré qu'une femme participant de la C 1. En revanche, on compte respectivement 15 et 12 membres de la deuxième et de la troisième cohorte parmi les femmes qui constituent notre étude. 
est très éprouvant [physiquement] ; avec toutes ces munitions, nous allions de Xakurke à Sehidan, puis de là à Shemzinan ; nous avions décidé de ne pas rester derrière ou de ne pas causer de problème parce que nous ne voulions pas que ces hommes nous critiquent. II s'agissait de notre fierté ; nous n'étions pas si conscientes [des questions de genre] à cette époque. Ferhat nous avait déjà menacées en nous confiant cette tâche. C'est ainsi que nous avons pris part [à notre première opération militaire]. \112

Dans le PKK, comme dans d'autres groupes sociaux virilistes et masculinistes, tels que les pompiers, la police, l'armée ou d'autres guérillas, la reconnaissance des femmes comme " égales » de leurs pairs repose sur la démonstration d'une implication supérieure aussi bien sur le plan physique, moral que d'investissement dans la lutte et la cause (voir Geesen, 2008 ; Pruvost, 2007 ; Pruvost et Cardi, 2012 ; Darley et Gauthier, 2018). Comme dans les groupes détenteurs de la violence légitime (armée, police), les femmes alignent leurs pratiques sur celles du groupe viril majoritaire et «apprennent que l'égalité s'acquiert par ressemblance au genre viril » (Pruvost, 2007, p. 97). Existe ainsi une intériorisation structurelle du combat entre les sexes au sein même du parti. La lutte contre le modèle patriarcal que les femmes ont souvent subi, au sein de leur famille, se perpétue dans ce cadre clandestin et est menée par elles parallèlement à la guerre contre l'État turc, à la lutte contre la forme État et contre la société (turque et patriarcale). Ces multiples fronts sont résumés par Yekta (G 2), dont la trajectoire est illustrative des obstacles rencontrées par les femmes engagées dans la C 2 : «Dans le parti aussi il y avait des restes de la phallocratie régnante. Autrement dit, il faut autant lutter contre la domination masculine que contre la domination de l'État. $»^{13}$

Ces témoignages et la réalité des rapports de genre au sein de la guérilla conduisent à nuancer, en particulier concernant les années 1990, l'image volontiers assumée par le parti qui voudrait que la participation des femmes à la guerre adoucisse cette dernière et contribue à développer une «éthique» de la guerre, voire une féminisation de cette dernière. L'alignement imposé des pratiques armées féminines sur celles du groupe viril majoritaire contribue à renforcer la parité mais ne transforme pas le rapport de force au sein du groupe détenteur des armes (Pruvost, 2007 ; Fusco, 2008)14. Les femmes n'y trouvent leur place qu'à condition d'adopter, dans un premier temps au moins et jusque dans les années 2000, l'ethos guerrier et les qualités qui définissent le bon guerrier mais dont la référence est d'abord masculine. Le modèle « professionnel » du combattant kurde est fondé sur la valorisation de la virilité au sens de la force, du courage et de la capacité à se battre - comme on le voit dans des corps militaires professionnalisés tels que la gendarmerie française (Pruvost, 2007). Le discours sur l'euphémisation de la guerre, qu'induirait la participation des femmes à la guerre, ne se déploie, dans le parti, qu'à partir des années 2000-2010 dans un contexte où le PKK est entré dans un processus de paix (Çandar, 2013), sans toutefois avoir renoncé aux armes et où il bénéficie d'un afflux remarquable de femmes vers ses rangs. Il participe d'un effort de

12 Berbiheyv (p. 11) explique la formation de « guitare, de saz, de mathématiques, de sciences de la vie, de géographie, etc. avec des enseignants venus de l'extérieur » ainsi que l'entraînement sportif que reçoivent les jeunes filles de 13 à 16 ans qui ont rejoint les YPJ.

${ }^{13}$ Cette reproduction des formes de domination a été documentée dans le cas des guérillas latinoaméricaines (voir Falquet, 1996, 2003 ; Boutron, 2019). Dans certains cas, les femmes s'appuient sur la non-mixité pour «agi[r] contre les violences genrées systémiques produites par l'État central (Iran, Turquie) et le patriarcat; contre celles produites par les hommes de leurs pays d'origine; et contre celles reproduites par leurs camarades hommes qui, avant de suivre les formations, arrivent en transportant les normes genrées apprises. » (Guillemet, 2017, p. 73)

${ }^{14}$ Le rôle des femmes-soldats dans les centres de torture d'Abu Ghraib démontre une incorporation féminine de «la domination virile (physique et sexuelle) que les soldats [...] ont toute latitude de déployer » (Cardi et Pruvost, 2012, p. 54). 
résolution de la contradiction entre un discours sur l'émancipation des femmes et le fait que le mimétisme guerrier qui s'impose aux femmes, loin de contester la prévalence virile, la conforte. La guérilla ne constitue pas un espace où il serait possible, pour les femmes d'« inventer de nouveaux rôles féminins alternatifs ». Il s'agit plutôt d'y « rationaliser les rapports de genre » et l'« économie libidinale »(Goodwin, 1997).

\title{
2. Une instrumentalisation des figures féminines par le parti ?
}

\author{
«L’une des ambitions inachevées de l'Önderlik ${ }^{15}$ est la réussite du mouvement des \\ femmes. » (Harun, engagé en 1994, C 2, rencontré en mars 2017)
}

De même que nous avons mis en évidence, en d'autres lieux (Guibet Lafaye, 2022b), un hiatus entre le développement de la théorie de la Femme libre et sa réception au sein de la population, susceptible de nourrir les rangs du PKK, on identifie un décalage entre le discours idéologique de promotion des femmes (voir supra) et les pratiques à l'œuvre dans le parti, au cours des années 1990 principalement mais aussi ultérieurement (voir Viyan, C $3{ }^{16}$ et Novellis, 2018). Le témoignage de Meddiyar, engagé en 1991 et rencontré en avril 2017, est sur ce point édifiant. Il évoque les premières années de la décennie 1990 :

«Certaines camarades devaient couvrir leurs cheveux. Tu ne pouvais pas parler librement aux camarades femmes, elles étaient un peu séparées de nous. Pendant une manifestation, ils plaçaient deux camarades femmes dans un groupe entièrement composé d'hommes. Pourquoi ? Parce que les femmes ne peuvent pas combattre. Elles ont des difficultés pour agir et lutter dans le conflit. Elles étaient protégées. Ainsi ils se limitaient à protéger la femme plutôt que de la libérer et de lui faire confiance. C'est-à-dire qu'il faut protéger le faible et lui apporter du soutien. Du genre : laisse-moi faire leur travail, laisse-moi porter sa charge, elles doivent être soutenues. C'était un sentiment entre pitié et protection. Ce n'était pas l'approche du parti mais où nous étions, c'était l'approche envers les femmes. »

À partir des vagues d'engagement massif des années 1990 au sein du parti, Öcalan produit plusieurs ouvrages (1992a, 1992b) constituant le cœur de ce que l'on a désigné la théorie de la Femme libre. Ils les enjoignent à développer une nouvelle personnalité, afin de se libérer des structures patriarcales qui les oppriment et de libérer de la sorte l'ensemble du peuple kurde. Pourtant les témoignages féminins et masculins font état d'une réticence persistante de la part des combattants face à l'entrée des femmes dans la guérilla durant cette décennie $(\mathrm{C} 2)$. On retrouverait dans des corps détenant le monopole de la violence légitime, comme la police ou l'armée, des obstacles de même nature (Pruvost, 2008), relatifs aussi bien à l'accès qu'à la promotion ultérieure des femmes. De la même façon qu'il est possible de mettre en évidence un écart manifeste entre la production des théories de la Femme libre et l'intégration du souci de l'émancipation des femmes dans les motivations des combattantes du PKK (Guibet Lafaye, 2022b) ${ }^{17}$, on peut souligner la prégnance du machisme au sein du parti durant cette décennie.

${ }^{15}$ Ce terme qui signifie, en turc, le chef, la direction désigne A. Öcalan.

16 Voir aussi les témoignages rapportés par le mouvement des femmes (https://jineoloji.org/en/2016/06/22/jineoloji-the-science-of-womens-liberation-in-the-kurdishmovement/).

${ }^{17}$ Soraya (C 2) qui a rejoint l'ERNK [le Front de Libération Nationale du Kurdistan] en 1991 à 24 ans est explicite : «En termes de stratégie au sein de la lutte, je n'avais pas vraiment conscience de la libération de la femme [au moment de m'engager], mais j'aimais ma patrie, j'aimais ces terres et j'en étais consciente. "Les Kurdes sont un peuple, ils ont leur pays et celui-ci peut seulement être sauvé par la lutte, nous entrons en guerre, il y a une guerre dans notre pays, nous allons le débarrasser de l'Etat 
Cet ethos se voit même reconnu par certains hommes, tels Meddiyar, Harun et Tekoşer, respectivement engagés pour les deux derniers en 1994 et 201318. Apparaît ainsi « un décalage évident entre l'image idéalisée de la combattante kurde "libérée" et la réalité des rapports de genre au sein de la guérilla du PKK. »(Grojean, 2013, p. 34 ; voir aussi Novellis, 2018, p. 130) Ce décalage est manifeste pour la C 2 (années 1990) mais il demeure jusque dans les années 2010, bien qu'alors les hommes qui continuent de s'étonner de la présence des femmes dans la guérilla gardent le silence sur le sujet et se conforment à la politique officielle du PKK concernant la libération des femmes (voir Tekoşer, interrogé en mars 201719). Les témoignages féminins, comme celui de Yekta (C 2), engagée en 1993 à 17 ans, permettent de mieux saisir que le transfert de ces luttes au sein de la guérilla :

«Dans la société kurde, dans la structure tribale, au foyer, il y a une domination masculine. Dans le parti aussi il y avait des restes de la phallocratie régnante. Autrement dit, il faut autant lutter contre la domination masculine que contre la domination de l'État. Nos camarades masculins n'étaient pas très convaincus de notre capacité à nous battre, en tant que femmes. C'était une première, non seulement au Kurdistan mais dans le monde. On dit toujours que seuls les hommes peuvent supporter des conditions de vie pénibles. Ces questions ont donné lieu à une lutte acharnée les premiers temps de mon engagement. Cette époque coïncidait avec un processus d'organisation d'unités de femmes combattantes. C'est à cette période que s'est constituée la première armée féminine. Des difficultés de ce genre ont perduré jusqu'en 1995. II y a l'exemple de la camarade Beritan, qui montrait que les femmes étaient désormais capables de mener leur propre guerre, d'établir et de tenir un campement quelque part. Son exemple renforçait cette conviction. À ce sujet aussi, il y avait une lutte. Ce mouvement a une morale, l'égalité homme-femmes, cela figure dans la philosophie de l'apoïsme. Pas question de discrimination. II n'y avait donc pas de résistance sérieuse pour s'opposer à la volonté des femmes. Les indicateurs étaient au vert pour que les femmes puissent réussir. 》

Alors même que des discriminations à l'encontre des femmes se perpétuent au sein du parti au cours des années 1990, fondées sur leur prétendue incapacité physique et militaire, s'instaurent progressivement des formes d'instrumentalisation de leur place, au sein de l'organisation, qui connaîtront leur apogée dans les années 2000-2010. Ces processus,

turc et des soldats turcs". C'était notre revendication. D'abord, nous allons libérer le Kurdistan, je dis ça en tant qu'individu bien sûr, je ne parle pas de la philosophie de l'Önderlik, "nous allons combattre en tant qu'individus, nous allons libérer notre pays, ensuite nous allons nous libérer". C'était une erreur bien sûr. Avec la lutte, nous avons appris à voir les choses selon plusieurs perspectives. C'était très différent en rejoignant la guérilla bien sûr, tu combats et tu découvres ta volonté et ta force, en tant que femme en société tu es dans une position de faiblesse, en besoin de protection, une victime, c'est différent au sein de la guérilla. Tu incarnes la force de volonté, tu es un être humain, une femme, une force, il n'y a rien que tu ne peux pas accomplir. C'est la différence entre la guérilla et la société. Tu es dans une position de faiblesse au sein de la société et ici tu es une force sur qui on peut compter et tu en es consciente. »

${ }^{18}$ Ils sont nés respectivement en 1970, 1973, 1991 et appartiennent donc pour les deux premiers à la C 2 et pour le dernier à la C 3 .

19 Tekoşer rappelle la prégnance des représentations patriarcales héritées : " C'est ma sœur, elle est plus jeune. Elle va travailler, elle va m'apporter un verre d'eau, une tasse de thé » mais souligne le silence de ses camarades masculins au nom du fait que «Même si nous ne comprenons pas, cela doit être correct!». Il reconnaît la difficulté, pour les hommes, de "donner un sens à l’idéologie de la libération des femmes, [parce] que nous ne sommes pas capables de la comprendre » et du fait de la conviction d'une «faiblesse des femmes ». En ce qui le concerne, il a dû désapprendre à vouloir porter les sacs de ses camarades féminines et reconnaître qu'au Rojava en particulier que les femmes jouent un rôle pionnier, sont devenues un sujet politique. 
participant de la production d'une image de soi du parti, s'appuient sur plusieurs facteurs. Il s'agit, en premier lieu, de la mythification de certaines femmes "mortes en martyrs » et, d'autre part, de la production d'une image de la femme détentrice de la violence clandestine à vocation civilisatrice et pacificatrice (voir infra 3.)20. Bien que Mazlum Doğan soit à l'origine de la dynamique sacrificielle, centrale au sein du $\mathrm{PKK}^{21}$, celle-ci se voit imputée de façon emblématique aux femmes à la suite de deux actions sacrificielles, menées par Berîtan et Zîlan. La première a été instituée en symbole de la résistance du PKK : le 25 octobre 1992, elle se jette d'une falaise plutôt que de se laisser capturer par l'armée turque. La seconde, Zîlan (Zeynep Kinanci) a mené une opération suicide le 30 juin 1996, en faisant exploser la bombe qu'elle portait sur elle au cœur d'un défilé militaire turc à Dersim, tuant et blessant des dizaines de soldats turcs ${ }^{22}$. Alors même que sur le terrain, la discrimination de la part de certains commandants et cadres continue de sévir, des femmes héroïques sont instituées en modèles, pour les guérillero/as, et investies d'une mission de libération (Çağlayan, 2012). La mise au premier plan de ces figures mythifiées participe à la fois de l'attractivité du groupe clandestin ${ }^{23}$ mais également de la transformation de l'image de soi du parti, «tueur de bébés » (Olga) selon l'État turc. Elle contribue à l'acceptabilité d'une violence politique toujours présentée et conçue comme défensive - donc légitime - en une violence qui, à partir des années 2000, est envisagée comme pacificatrice voire civilisatrice. Or la valorisation des combattantes joue un rôle majeur dans cette transformation de l'image de soi du parti. Le témoignage de Viyan (C 3) sur la place des femmes, au sein du mouvement, lors de son engagement en 2009 est, sur ce point, éclairant :

« La période à laquelle j'ai rejoint [le PKK] n'est pas si lointaine, [mais] elle ne date pas d'hier; c'était il y a environ neuf ans. Jusque là, les femmes avaient déjà développé leur lutte au sein du PKK de manière significative : l'idéologie de la libération des femmes avait déjà été déclarée, et les femmes avaient déjà obtenu leur propre et authentique "parti-fication" et "armi-fication". Ces réussites ont précédé mon engagement et avaient déjà suscité au PKK un véritable sens de la liberté. Mais ce sens a aussi été renouvelé avec les nouveaux engagements car les individus sont en quête de certaines choses. Tout le monde ne s'engage pas avec le même sentiment de liberté ou avec le même point de vue. C'est en fait quelque chose qui se perpétue, les gens se renouvellent, leurs points de vue idéologiques changent, la manière d'aborder la liberté et la lutte change en permanence. Bien sûr, tu peux voir avec le temps les avancées de la lutte des femmes. D'accord, nous parlons d'une organisation qui s'est peut-être dotée de sa propre armée, de son propre parti et qui mène une activité contestataire sur le front, mais de nombreuses femmes se sont engagées en particulier après 20I0. Par exemple, c'est la même chose de nos jours et ces dernières années. C'est lié à une quête spécifique et à la réponse apportée par le PKK à celle-ci. La place de la femme... On peut dire que la femme qui combat au PKK représente un exemple pour le monde en termes de mouvement de libération de la femme, notamment après la formation des YPJ et de l'action à Kobané en 20 I4. C'est mon constat. Bien sûr il y a une évolution quotidienne, en ce moment les femmes ont leur propre armée au Rojava, au Şengal, au Bakûr, je parle du YPG, du YPJ, du YPS-Jîn, du JBŞ, du YJŞ, leurs progrès ici sont très importants. »

\footnotetext{
${ }^{20}$ Voir infra 3.

${ }^{21}$ Il fut l'un des fondateurs du PKK. Le 21 mars 1982, date du nouvel an kurde (Newroz), il se suicide pour protester contre les conditions de détention et la répression (torture) dans la prison $n^{\circ} 5$ de Diyarbakır.

${ }^{22}$ Voir la mémoire qu'en garde Lilas.

${ }^{23}$ Voir Novellis, 2018, p. 129.
} 
Se présenter et se promouvoir comme parti des femmes est impératif pour le PKK, qui poursuit un objectif de légitimation. L'image de soi ainsi produite temporise son inscription sur la liste des organisations terroristes de l'Union européenne. Pourtant, y compris après les années 1990 et aujourd'hui encore, persiste une ambivalence entre cette image de soi et ce que vivent certaines cadres et sympathisantes au sein du parti. L'étude générationnelle souligne l'évolution et la persistance de cette dualité dans le temps long. Des hommes (comme Tekoşer) aussi bien que des femmes de la C 3 (telles Rojen et Berbiheyv) font état de la reproduction de formes de sexisme voire de discrimination. Ces attitudes se maintiennent alors même que l'accès à la violence politique, l'« armification » des femmes a été conçue par A. Öcalan comme vecteur de leur émancipation (voir supra).

Le parti réécrit rétrospectivement son histoire au prisme de la lutte de libération des femmes et en l'ancrant dans le mythe d'un néolithique matriarcal. Pourtant, d'une part, l'organisation strictement paritaire des instances du mouvement n'a pas toujours suffi à disqualifier les attitudes portées par les stéréotypes de genre. D'autre part, on peut s'interroger sur les formes d'instrumentalisation sous-jacentes à la mise en avant des femmes combattantes, dans la construction d'une image et d'une présentation de soi du parti sur le plan international. Certaines femmes de la C 2, qui ont subi les difficultés d'intégration dans les années 1990, en sont conscientes. Shilan ne manque pas d'évoquer la façon dont les cadres masculins du parti mettent en avant la lutte des femmes :

« Heval Cuma a fait une déclaration le 8 mars, il a dit "si le PKK a réussi à se maintenir encore aujourd'hui c'est grâce à l'Önderlik et à la valeur que notre organisation a accordé aux femmes". II a continué en disant "ce mouvement est un mouvement de femmes". Tu connais Adem Heval en Europe. II a dit une chose similaire "si ce n'était pas pour les femmes, peutêtre que le PKK n'aurait pas pu atteindre ce niveau". Le PKK n'aurait pas pu autant s'agrandir. La lutte des femmes protège le PKK de l'oppression de tous les autres États. Bien sûr le leader Apo a assuré ça dès le début. Puis est venu le mouvement des femmes. »

Dans ce mouvement de guérilla kurde, comme ce peut être le cas au sein des armées régulières ou de polices contemporaines occidentales, la féminisation de ces corps ainsi que les femmes sont considérées comme des ressources pouvant être instrumentalisées. La complémentarité des sexes n'échappe pas à une logique utilitariste. Les groupes clandestins, qu'il s'agisse du PKK, des YPG, des YPJ ou des collectifs apparentés, mobilisent l'imaginaire des femmes héroïques pour la propagande interne et externe et l'image de soi que ces organisations veulent diffuser d'elles-mêmes. L'appropriation du discours de l'émancipation par les combattantes, au fil des années passées dans la guérilla (Guibet Lafaye, 2022b), contribue à une évolution de leur appréhension des rapports de genre et de leur implication dans leur transformation vers un niveau accru d'égalité des sexes. Néanmoins l'organisation pyramidale et fortement structurée de ces groupes politiques n'exclut pas que les dirigeants politiques (hommes ou femmes) puissent «utiliser les femmes et leur corps de manière pragmatique pour faire avancer leurs propres intérêts » (Gökalp, 2010, p. 563) ou ceux de leur organisation. Jusqu'au début des années 2000 au moins, l'acceptation et la progression des femmes au sein de la guérilla n'ont pas été synonymes d'une réelle transformation des rapports sociaux de sexe, en dépit du projet politique affichée ${ }^{24}$. Il ne s'est pas non plus accompagné d'un bouleversement de ces rapports sur un plan social plus large ${ }^{25}$.

${ }^{24}$ Sur la question du partage effectif et égalitaire des tâches militantes au sein de la guérilla, voir Solina, 1997 ; Flach, 2003, 2007 ; Grojean, 2013. On observait jusque dans les années 1990 qu'« un certain nombre de clichés liés aux rôles sociaux féminins ont continué de dominer les rapports sociaux, s'apparentant à une véritable "division sexuelle du travail révolutionnaire" (Falquet, 2003): éloignement des combats, tendance à croire que les femmes sont davantage susceptibles de "trahir", 


\section{Le féminisme martial}

La constitution de groupes de combattantes et l'intégration des femmes à la guérilla porte la littérature académique à évoquer l'existence d'un «féminisme martial » (Schalk, 1994 ; Gayer, 2019; Grojean, 2019) dont il convient néanmoins d'interroger les contours et la réalité. Certaines femmes, parmi les plus jeunes notamment, investissent la violence politique comme un vecteur d'affirmation de soi et un moyen privilégié de leur émancipation. Tel est le cas de Rojen (C 3) qui offre un rare exemple de féminisme martial :

« Après que les femmes ont rejoint le PKK, elles ont développé l'idée sur cette base. Aujourd'hui, à Kobanî, si une femme de 60 ans, une femme de 50 ans défend son pays, établit son parlement, ce sont les idées de I'Önderlik [Öcalan] qui l'ont permis. Le PKK l'a fait [...]. II a créé cette idée. Qu'est-ce que ça veut dire une armée de femmes? Quelle était la différence avec l'État? Dans celui-ci, "l'homme est l'armée, le travail de la femme est de cuisiner, d'élever les enfants." [...] Par exemple après le processus de 1992 une armée de femmes est établie au sein du PKK, comme le YJA-Star. Ainsi vous avez acquis votre identité idéologiquement et en tant qu'armée. Cette fois, l'État ne peut pas faire cette justification ${ }^{26}$. Lorsque vous devenez une armée, vous apprenez réellement à vous connaître. Vous révélez votre pouvoir. Si je n'avais pas rejoint le PKK, je serais une femme normale, je veux dire que je ne connaitrais personne. $\mathrm{Si}$ je ne me battais pas, je n'aurais même pas le droit à la parole. Mais si je parle aujourd'hui, ce que je vis actuellement est le résultat de mon expérience dans les montagnes [i.e. dans la guérilla]. »

L'investissement de ce rôle de combattante participe d'une "transformation identitaire », caractéristique d'un processus d'«alternation » (Berger et Luckmann, 1966), qui illustre une césure nette entre l'identité préalable et celle du « converti » entré dans le mouvement. Une double dynamique, microsociale (individuelle) et mésosociale (collective) est à l'œuvre dans cette production de soi identitaire, dans la production de cette nouvelle identité. En effet, l'identité sociale advient comme « le résultat à la fois stable et provisoire, individuel et collectif, subjectif et objectif, biographique et structurel, des divers processus de socialisation qui, conjointement, construisent les individus et définissent les institutions » (Dubar, 1996, p. 111). En ce sens, l'identité est la résultante, à un moment donné, du processus de socialisation d'un individu en même temps qu'elle informe son action, comme le suggère le témoignage de Rojen. Au cours de cette évolution, certains traits de l'identité sociale, i.e. certains rôles sociaux composant l'identité sociale, peuvent être plus ou moins saillants. Ces derniers se structurent selon un principe d'organisation hiérarchique (Stryker et al., 2000), lié aux conditions contextuelles de leur acquisition, de leur expression et de leur reconceptualisation ${ }^{27}$ (Fillieule et al., 2010, p. 215).

Sur ce plan, c'est-à-dire aussi bien sur le plan des conditions pratiques ou contextuelles que sur le plan conceptuel, l'existence de structures déjà constituées, telles que le YJA-Star (strate

etc. » (Grojean, 2019, p. 152).

${ }^{25}$ On parviendrait à la même conclusion en considérant « la production du genre » au sein du Sentier Lumineux et le cas du Pérou (Felices-Luna, 2019). Des conclusions similaires ont été établies pour l'Afrique du Sud et l'Algérie (voir Bucaille, 2019).

${ }^{26}$ I.e. " L'homme est l'armée, le travail de la femme est de cuisiner, d'élever les enfants. »

${ }^{27}$ Sur ce plan, les théories d'Öcalan (1992a, 2014) puis la finnelojî, dont l'enseignement est dispensé dans le mouvement kurde et la guérilla, ont joué un rôle de premier ordre dans la saillance d'une identité émancipée ou tendant vers l'émancipation de ces femmes. 
méso-sociologique ${ }^{28}$, joue un rôle décisif dans la possibilité d'une réappropriation et d'un investissement subjectifs (niveau microsocial) du féminisme martial. Comme nous l'avons rappelé, les premières divisions militaires non-mixtes se constituent au début des années 1990 au sein du PKK. À partir des années 2000, le parti promeut une « égalité dans la différence » (Jineoloji Akademisi, 2015). Il assume un discours selon lequel la «Kurdicité » originelle, pendant la période néolithique, serait fondée sur un équilibre entre «matriarcat » [pouvoir des femmes], «socialisme primitif » [démocratie directe] et «nature » [l'écologie] (Öcalan, 2014, p. 16). La Finelojî, science des femmes et de la vie libre, a progressivement été instituée en discipline dispensée par le mouvement des femmes ${ }^{29}$. Le terme, dérivé de jin (signifiant femme en kurde) et de logos, a été utilisé pour la première fois par le PKK en 2008 (Çağlayan, 2020, p. 78) afin de développer une critique de la domination masculine et contribuer à l'autonomisation des femmes. Cette «science de la modernité démocratique » est fondée sur cinq principes ${ }^{30}: 1$. Welatparezi, consistant dans le refus de l'éloignement, du colonialisme et de l'assimilation imposés aux femmes; 2. la libre pensée ou autonomie de jugement : les femmes doivent prendre leurs propres décisions et rompre mentalement avec les structures qui les dominent ; 3. l'organisation autonome des femmes, conformément à l'idée que le patriarcat ne sera vaincu que si les femmes ont la possibilité de s'organiser elles-mêmes; 4. la lutte pour le changement, dans la mesure où il ne s'agit pas simplement d'exiger des choses de l'oppresseur mais de conquérir des droits par la lutte et de créer des alternatives; 5. l'esthétique et l'éthique, considérant que les femmes ne doivent pas s'en tenir aux modèles de beauté dictés par la société ou par les hommes ${ }^{31}$.

Ce cadre idéologique suscite de nouveaux «arrangements de genre » (Roux et al., 2005, p. 7), où l'égalité est érigée en fondement de la «Kurdicité ». La «Révolution de la femme » repose sur l'organisation en non-mixité et la finnelojî (Guillemet, 2017, p. 69). Cette organisation est présentée " comme un outil et une condition de l'émancipation des femmes. » (Öcalan, 2014 ; Jineoloji Akademisi, 2015) ${ }^{32}$. La strate méso-sociale contribue donc à la dynamique individuelle (microsociologique) d'alternation. Elle participe de la production d'une «structure de plausibilité » (Berger et Luckmann, 1966), i.e. du déploiement et de l'adhésion à une représentation du monde, à un «cadrage » (Benford et Snow, 2000, p. 614) des conditions objectives auxquelles l'acteur fait face, permettant de faire durer la « conversion » (Renou, 2009). Cette conversion subjective consiste à la fois dans un processus de transformation «quasi-totale» de la réalité subjective de l'individu, celui-ci «devenant autre », en même temps qu'il adhère à un « nouveau monde » subjectif (Berger et Luckmann, 1966 ; Guibet Lafaye et Rapin, 2017). Dans le cas présent, la dynamique de transformation identitaire conduit à la redéfinition d'une identité constitutive d'un engagement total (Yon, 2005).

${ }^{28}$ Voir supra le témoignage de Berbiheyv (note 12).

${ }^{29}$ Le gouvernement autonome du Rojava l'a incluse dans ses programmes éducatifs officiels (Çağlayan, 2020, p. 78).

30 https://jineoloji.org/en/2016/06/22/jineoloji-the-science-of-womens-liberation-in-the-kurdishmovement/

${ }^{31}$ Cette «nouvelle science » critique le lien entre hégémonie, oppression et sciences sociales, en proposant de reconsidérer l'écriture de l'histoire, excessivement marquée par l'hégémonie masculine et l'invisibilisation de l'histoire des femmes. « La Jinéologie veut réinterpréter la mythologie, la religion, la philosophie et la science. Elle veut retrouver les traces et la vérité des femmes afin de trouver les voies de la libération des femmes. Par cette libération, elle vise la libération de la société entière et l'établissement d'une vie libre et communautaire. [...] La Jinéologie s'emploie à créer une transformation, non seulement en modifiant l'autodéfinition de la femme, mais aussi en critiquant l'État, les systèmes de pouvoir, leurs institutionnalisations et la mentalité formée autour d'elles. » (https://jineoloji.org/en/what-is-jineoloji/)

32 Voir Rostampour, 2020, 2021. 
Néanmoins l'identification à ce féminisme martial doit être nuancé, dans la mesure où les femmes s'approprient plus volontiers la naturalisation du féminin portée par la théorisation du leader qui l'associe à la paix (Öcalan, 1992, 2013; Koç, 1993, p. 37-39). Depuis son arrestation en 1999, Öcalan déploie une idéologie promouvant un «Combat pour la civilisation », c'est-à-dire la démocratie, la paix et l'écologie, considérées comme les qualités intrinsèques de la «Femme libre » (Grojean, 2013, p. 26). Nombreuses sont les combattantes à considérer le recours aux armes comme une nécessité dans le contexte du Moyen-Orient au même titre que les combattants - mais elles reconnaissent préférer n'avoir jamais eu à les utiliser $^{33}$. Cette naturalisation traverse le discours de Shilan (C 2), engagée en 1992 à 17 ans, qui tisse dans un même propos ce paradigme avec celui de la violence politique comme vecteur de l'émancipation féminine :

« II n'y a pas de guerre dans la nature de la femme, c'est entièrement pacifique, néanmoins au PKK, du point de vue du PKK, la guerre est aussi une question de paix. Par exemple, comme la formule "un lion est un lion, peu importe qu'il s'agisse d'un homme ou d'une femme". [...] La guerre s'impose comme une obligation pour la libération de la femme de la domination masculine. Aujourd'hui des milliers de femmes suivent le chemin suivi par les héroïnes comme Zîlan et Berîtan. Des milliers de femmes suivent la voie montrée par ces héroïnes. En fait la guerre est un instrument qui brise l'esclavage des femmes dans la société. II brise ces chaînes. Autrement dit, la nature féminine est plus proche de la paix que de la guerre. $»^{34}$

La production d'une nouvelle identité ne s'opère pas tant autour du féminisme martial comme usage d'une violence politique défensive - donc légitime -, voire autour de la défense de la communauté kurde, qu'en référence à un projet d'émancipation. La parole des combattantes permet ainsi de nuancer la place du féminisme martial dans l'image de soi qu'elles produisent et assument. En revanche, l'approche académique semble influencée par le surinvestissement médiatique des guérilleras kurdes depuis les années 2000 et surtout 2010 dans la lutte contre l'État islamique (Begikhani et al., 2018), ainsi que le témoignage de Viyan précédemment évoqué le suggère (note 33). Les expériences de construction de soi ne s'articulent donc pas simplement, du côté féminin, en référence à l'usage de la violence politique. Les femmes se construisent comme «sujets » politiques et parties prenantes d'une identité politique collective (Guillemet, 2017, p. 75), au-delà du recours aux armes et de tout féminisme martial.

33 Viyan (C 3) l'exprime sans détour : «Avant de m'engager, je pensais que la violence n'avait pas sa place dans la vie humaine. Mais la légitime défense est une chose différente selon moi. Quand je pense à la violence et à la guerre, je me considère comme quelqu'un qui ne pourrait jamais y prendre part, et j'avais seulement une seule question en tête avant de m'engager: "Puis-je prendre les armes et combattre, puis-je blesser quelqu'un ?". Non seulement moi, mais beaucoup dirait ça, mais les femmes sont plus susceptibles de le dire. La majorité des camarades femmes diraient la même chose. La guerre est un point de rupture pour nous. Parce que malheureusement, les femmes sont celles qui portent la responsabilité et la douleur de la guerre par la suite. Mais tu peux te convaincre qu'il s'agit de légitime défense, ce n'est qu'une question de légitime défense. »

${ }^{34}$ On retrouverait des arguments comparables dans le discours de Çiçek (C 2) : «Vous savez, si cela dépendait de moi, les gens ne mourraient pas du tout [ne seraient pas tués]. Je suis contre la violence en tant qu'être humain, en tant que femme. » 


\section{Conclusion}

À partir de données de terrain, nous avons souligné les limites de l'imposition hiérarchique d'une idéologie d'émancipation et de transformation des rapports de genre, y compris dans une organisation structurée de façon pyramidale. La promotion de cette évolution normative se heurte à l'importation de normes et de représentations dites traditionnelles concernant la division genrée du travail, y compris dans l'espace révolutionnaire (Falquet, 2003). Le PKK n'est pas le seul à s'être distingué en la matière. D'autres exemples existent au Moyen-Orient (cas de l'Iran: Shahidian, 1997 ; Guillemet, 2017) ou en Amérique latine (Boutron, 2015 ; Falquet, 1996, 2003 ; Felices-Luna, 2008). L'évolution des rapports de genre est loin de seulement dépendre de l'impulsion idéologique du leader mais est également le fruit des "rapports de force maintenus par les combattantes au sein de leurs propres partis » (Guillemet, 2017, p.66) et luttant contre la domination socio-structurelle extérieure et intérieure.

Si la littérature académique a produit quelques travaux sur les rapports de genre au sein de la guérilla kurde (Solina, 1997; Flach, 2003, 2007), il demeure très délicat d'étudier empiriquement la perpétuation des dominations de genre, dans un contexte où coexistent une « sensibilisation à la domination masculine et aux sentiments d'incompétence féminine » ainsi qu'« une asexuation/désexualisation des militants et combattants » (Grojean, 2019, p. 153). Ce que permettent l'observation in situ ou les démarches ethnographiques de terrain n'est pas toujours saisissable dans l'entretien semi-directif, c'est pourquoi nous avons tenté de confronter les paroles des enquêté.e.s à d'autres sources. Les femmes restées dans la guérilla adhèrent souvent à un discours d'émancipation qui leur confère la part belle. Elles se l'approprient et le mobilisent dans le jeu de leur construction identitaire et de l'affirmation de soi comme sujet politique, émancipé des rapports sociaux de genre. Dès lors, il peut être approprié de se mettre également à l'écoute des voix masculines, en particulier lorsque certains reconnaissent que la perpétuation du patriarcat au sein du parti a pu remettre en question leur engagement dans la guérilla, au début des années 1990, mais aussi de la parole des femmes désengagées du parti, revenant sur leurs trajectoires d'engagement et les conditions de ce dernier.

Enfin, l'approche par le féminisme martial pourrait bien constituer un écueil pour aborder la question de l'émancipation de femmes qui ont pris l'option armée. Non seulement celle-ci peut être un choix induit par le fait que, dans les années 1990, voire ultérieurement dans des sociétés conservatrices et patriarcales, le seul modèle de vie qui s'offre à elles est celui défini par les stéréotypes de genre assignant les femmes à des rôles traditionnels. En outre, ce serait une erreur de conférer à l'usage des armes une fonction centrale, dans la logique émancipatrice, sans tenir compte des formes parallèles d'institution de soi comme sujet politique. La réflexion académique doit se garder du piège médiatique consistant à fétichiser les combattantes - kurdes ou d'autres origines - en dissociant leur rôle militaire de leur investissement et de leur formation politiques. Les femmes se battent «aussi bien avec des idées qu'avec des balles » (Dirik, 2015) pour un projet émancipateur qui ne concerne pas seulement les femmes.

Plus fondamentalement, il importe de s'interroger sur les incidences, les conséquences et les effets du «féminisme martial» sur l'évolution des rapports de genre dans des sociétés patriarcales voire sur le destin de l'émancipation des femmes plus largement et son analyse. Conférer à l'usage des armes une fonction centrale sur ce dernier reconduit la valorisation d'un «comportement atypique pour les femmes » (Toivanen et Baser, 2016, p. 306) et peut mettre en difficulté des formes d'émancipation passant par d'autres voies ainsi que leur promotion dans un contexte social plus large. Sans cette précaution, on perpétuera le fait que 
l'accès des femmes à la violence politique ne peut transformer l'état des rapports sociaux de sexe.

\section{Références}

Allison C., "Folklore and Fantasy: the Presentation of Women in Kurdish Oral Tradition ", in S. Mojab (ed.), Women of a Non-State Nation. The Kurds, Costa Mesa, Mazda Publishers, 2001, p. 181-194.

Begikhani Nazand, Wendelmoet Hamelink et Nerina Weiss, "Theorising women and war in Kurdistan: A feminist and critical perspective », Kurdish Studies, vol. 6, n 2, May 2018, p. 530 .

Benford R.D. et D.A. Snow, «Framing Processes and Social Movements: An Overview and Assessment », Annual Review of Sociology, vol. 26, 2000, p. 611-639.

Berger Peter et Thomas Luckmann, La construction sociale de la réalité [1966], Paris, Meridiens-Klincksieck, 1986.

Boutron Camille, "Women at war, war on women : reconciliation and patriarchy ", in Seema Shekhawat, Female Combatants in Conflict and Peace, Hampshire, Palgrave Macmillan, 2015, p. 149-166.

Boutron Camille, «De la subversion à l'instrumentalisation. Trajectoires combattantes féminines et réaffirmation du patriarcat dans le Pérou de l'après-conflit », in Caroline Guibet Lafaye et Alexandra Frénod (dir.), S'émanciper par les armes? Sur la violence politique des femmes, Paris, Presses de l'Inalco, 2019.

Bucaille Laetitia, «Louisette et Dipuo, combattantes du FLN et de l'ANG. Engagement et transgression ", in Caroline Guibet Lafaye et Alexandra Frénod (dir.), S'émanciper par les armes? Sur la violence politique des femmes, Paris, Presses de l'Inalco, 2019.

Çağlayan H., «From Kawa the Blacksmith to Ishtar the Goddess: Gender Constructions in Ideological-Political Discourses of the Kurdish Movement in post-1980 Turkey », European Fournal of Turkish Studies [Online], 14 | 2012, http://journals.openedition.org/ejts/4657

Çağlayan H., « Kurdish Women as Political Agents: Kurdish Political Movement, Gender Equality, and Women's Freedom ", in Women in the Kurdish Movement. Palgrave Macmillan, Cham, 2020.

Çandar C., "Öcalan's Message is Much More Than a Cease-Fire », Al-Monitor, 24/03/2013, http://www.al-monitor.com/pulse/originals/2013/03/ocalan-ceasefirenewroz-speech-farewell-to-arms.html, consulté le 10/5/2021.

Cardi Coline et Geneviève Pruvost (dir.), Penser la violence des femmes, Paris, La Découverte, 2012.

Darley Mathilde et Jérémie Gauthier, «Bon flic bon genre. L'ordre des sexes dans la police ", in Jérémie Gauthier et Fabien Jobard (dir.), Police: Questions sensibles, Paris, PUF, 2018, p. 77-93.

Dirik D., «The Women's Revolution in Rojava: Defeating Fascism by Constructing an Alternative Society », in Strangers in a Tangled Wilderness (eds.), A Small Key Can Open A Large Door: The Rojava Revolution, Wilderness, Combustion Books, 2015, p. 55-64.

Dubar, Claude, La socialisation. Construction des identités sociales et professionnelles, Paris, Armand Colin, 1996.

Falquet Jules-France, «Entre rupture et reproduction: femmes salvadoriennes dans la guerre révolutionnaire (1981-1992)», Nouvelles Questions Féministes, vol. 17, n 2, 1996, Paris, p. 5-38. 
Falquet J., «Division sexuelle du travail révolutionnaire: réflexions à partir de la participation des femmes salvadoriennes à la lutte armée (1981-1992) », Cahiers d'Amérique latine, $\mathrm{n}^{\circ} 40,2003$, p. 109-128.

Felices-Luna M., « Déviance et politique : la carrière des femmes au sein de groupes armés contestataires », Déviance et Société, vol. 32, n 2, 2008, p. 163-185.

Felices-Luna M., «Sentier Lumineux et la production du genre: discours réducteur, pratiques inattendues, expériences disparates ", in Caroline Guibet Lafaye et Alexandra Frénod (dir.), S'émanciper par les armes? Sur la violence politique des femmes, Paris, Presses de l'Inalco, 2019.

Fillieule Olivier, Éric Agrikoliansky et Isabelle Sommier, Penser les mouvements sociaux conflits sociaux et contestations dans les sociétés contemporaines, Paris, La Découverte, 2010.

Flach A., Fiyanekê din - ein anderes Leben. Zwei Jahre bei der kurdischen Frauenarmee, Köln, Mesopotamien Verlag, 2003.

Flach A., Frauen in der kurdischen Guerilla. Motivation, Identität und Geschlechterverhältnis in der Frauen Armee der PKK, Köln, Pappy Rossa Verlag, 2007.

Fusco Coco, Petit manuel de torture à l'usage des femmes-soldats, Montréal, Lux Éd., 2008.

Gayer L., «Militariser les femmes. Doctrines, pratiques et critiques du féminisme martial en Asie du Sud ", in Caroline Guibet Lafaye et Alexandra Frénod (dir.), S'émanciper par les armes? Sur la violence politique des femmes, Paris, Presses de l'Inalco, 2019.

Geesen A., Une femme d'honneur au miroir des faits : la féminisation de la gendarmerie (1983-2005), Vincennes, Service historique de la Défense, 2008.

Goodwin Jeff, "The Libidinal Constitution of a High-Risk Social Movement: Affectual Ties and Solidarity in the Huk Rebellion, 1946 to 1954 », American Sociological Review, vol. 62, $\mathrm{n}^{\circ} 1,1997$, p. 53-69.

Gökalp D., «A Gendered Analysis of Violence, Justice and Citizenship: Kurdish Women Facing War and Displacement in Turkey », Women's Studies International Forum, vol. 33(6), 2010, p. 561-569.

Grojean Olivier, «Théorie et construction des rapports de genre dans la guérilla kurde de Turquie », Critique internationale, vol. 60, $\mathrm{n}^{\circ}$ 3, 2013, p. 21-35.

Guibet Lafaye C., "Évolution générationnelle des facteurs de l'engagement au PKK », Revue SociologieS, Toulouse, Université Jean Jaurès, 2022a. [À paraître.]

Guibet Lafaye C., «S'engager au PKK pour la libération des femmes? Lecture critique d'une construction politique ", Confluences méditerranéennes, Paris, L'Harmattan, 2022b. [À paraître.]

Guibet Lafaye C. et A.-J. Rapin, «Individualisation et dépolitisation d'une notion : évolutions contemporaines de la sémantique de la radicalisation ", Politiques de communication, $\mathrm{n}^{\circ}$ 8, mai 2017, PUG, Grenoble, p. 127-153.

Guibet Lafaye Caroline et Barish Tugrul, «PKK militants: from the 1970s to nowadays. Three cohorts », Terrorism and Political Violence, 2022. [À paraître.]

Guillemet Sarah, "'S'organiser au maquis comme à la ville". Les femmes kurdes au Comité des révolutionnaires du Kurdistan Iranien (Komala) et au Parti des Travailleurs du Kurdistan (PKK) », Confluences Méditerranée, n 103, 2017, p. 65-79.

Jongerden Joost, "Gender equality and radical democracy: Contractions and conflicts in relation to the "new paradigm" within the Kurdistan Workers' Party (PKK) », Anatoli [En ligne], 8 | 2017, mis en ligne le 22 mars 2018, consulté le 20 février 2020.

Kergoat D., "Division sexuelle du travail et rapports sociaux de sexe », in H. Hirata, F. Laborie, H. Le Doare et H. Senotier (dir.), Dictionnaire critique du féminisme, Paris, PUF, 2000, p. 35-44.

King D. E., "The Personal is Patrilineal: "Namus" as Sovereignty », Identities. Global Studies in Culture and Power, vol. 15(3), 2008, p. 317-342.

KJK, https://www.kjk-online.org/?lang=en, 2013, consulté le 10/5/2020. 
Laperrière A., "Les critères de scientificité des méthodes qualitatives », in J. Poupart, J.-P. Deslauriers, L.-H. Groulx, A. Laperrière, R. Mayer et A. P. Pires (dir.), La recherche qualitative : enjeux épistémologiques et méthodologiques, Boucherville (Québec), Gaëtan Morin éd., 1997.

Novellis Andrea, "The Rise of Feminism in the PKK: Ideology or Strategy? », Zanj: The Fournal of Critical Global South Studies, vol. 2, nº 1, Pluto Journals, 2018, p. 115-133.

Öcalan Abdullah, Kadin ve Aile Sorunu (Ed. S. Erdem) (Woman and Family Question), Istanbul, Melsa Yayınları, 1992a.

Öcalan Abdullah (pseud. Ali Frrat), Kürdistan'da Kişilik Sorunu (The question of personality in Kurdistan), Istanbul, Melsa Yayınları, 1992b.

Öcalan Abdullah, Kurdistan Report, n 18, Mai 1993, p. 22-26.

Peteet Julie, Gender in crisis: women and the palestinian resistance movement, New York, Columbia University Press, 1991.

Pruvost G., Profession : policier ; Sexe : féminin, Paris, MSH, 2007.

Pruvost G., De la "sergote » à la fermme flic : Une autre histoire de l'institution policière (1935-2005), Paris, La Découverte, 2008.

Renou G., «La découverte ou l'ignorance. Contribution à l'étude des opérateurs d'alternation dans la formalisation des identités militantes ", in B. Voutat, M. Surdez, M. Voegtli (dir.), Identifier - s'identifier. À propos des identités politiques, Antipodes, Lausanne, 2010.

Schalk Peter, "Women Fighters of the Liberation Tigers in Tamil İlam: The Martial Feminism of Atēl Palacinkam », South Asia Research, vol. 14, n² 2, 1994, p. 1-22.

Serok M., Kadının toplumsal sözleşmesi, İstanbul, Hevi Yayınları, 2001.

Snow D.A. et Machalek R.A., "The Sociology of Conversion », Annual Review of Sociology, vol. 10, 1984, p. 167-190.

Stryker Sheldon, Timothy Owens et Robert White (dir.), Self, Identity, and Social Movements, Minneapolis, University of Minnesota Press, 2000.

Solina Carla, Der Weg in die Berge. Eine Frau bei der kurdischen Befreiungsbewegung, Hambourg, Nautilus, 1997.

Toivanen M. et B. Baser, «Gender in the Representations of an Armed Conflict. Female Kurdish Combatants in French and British Media», Middle East Fournal of Culture and Communication, vol. 9, 2016, p. 294-314.

Weiss N., "Gender Roles », in S. Maisel (ed.), The Kurds: An Encyclopedia of Life, Culture and Society, Santa Barbara, ABC-CLIO, 2018.

Yon K., «Modes de sociabilité et entretien de l'habitus militant. Militer en bande à l'AJSOCI », Politix, n 70, 2005, p. 137-167.

Yüksel Metin, «The Encounter of Kurdish Women with Nationalism in Turkey », Middle Eastern Studies, vol. 42, n 5, 2006, p. 777-802.

\section{Annexe}

L'enquête de sociologie qualitative qui nourrit cette analyse a été réalisée entre mars 2016 et mars 2017 par une équipe de recherche, composée de Caroline Guibet Lafaye, Barish Tugrul et Neslihan Yaklav. Nous avons interrogé 64 militant.e.s appartenant ou ayant appartenu au PKK. Tous sont originaires de Turquie. Nous avons privilégié ce choix méthodologique afin d'avoir un groupe de répondants issus du même contexte macrosocial et historique. Les personnes ont été contactées soit directement, en particulier celles qui avaient quitté le parti, soit par la méthode «boule de neige»(Laperrière, 1997). En dépit des difficultés propres à ce type de terrain, nous avons veillé à préserver une distribution équilibrée en termes d'âge, de sexe et d'origine géographique des acteurs. Ces derniers sont nés entre 1955 et 1991. Les entretiens ont été enregistrés et entièrement transcrits et traduits. 
Ils ont été menés sur les lieux de vie ou d'activité des militant.e.s (Irak) et ont duré entre 42 minutes et plus de 3 heures, pour une moyenne de 76 minutes par entretien. Tous ont été menés en face-à-face, en turc ou en kurde (Kurmanji). La collecte de données primaires et l'utilisation des archives du parti, notamment les deux revues mensuelles Serxwebûn et Berxwedan ${ }^{35}$, ont été combinées avec une étude des sources contemporaines, la consultation de documents gouvernementaux et non-gouvernementaux et de toute source sur le sujet en anglais, français et turc.

\begin{tabular}{|c|c|c|c|c|c|c|c|}
\hline Pseudonyme & $\hat{A g e}$ & Cohorte & $\begin{array}{c}\text { Années } \\
\text { d'engagement }\end{array}$ & $\begin{array}{l}\text { Age d'entrée } \\
\text { dans le parti }\end{array}$ & $\begin{array}{c}\text { Année de } \\
\text { sortie du } \\
\text { parti }\end{array}$ & $\begin{array}{l}\text { Date de } \\
\text { l'entretien }\end{array}$ & $\begin{array}{c}\text { Lieu de } \\
\text { l'entretien }\end{array}$ \\
\hline Sarah & 50 & C 1 & 1989 & 23 & 2004 & août 2016 & Erbil \\
\hline Bese & 48 & C $1 / \mathrm{C} 2$ & 1989 & 21 & & 2016 & Kandil \\
\hline Soraya & 49 & C 2 & 1991 & 24 & & août 2016 & Kandil \\
\hline Dicle & 46 & C 2 & 1993 & 23 & 2000 & août 2016 & Erbil \\
\hline Mizgin & 44 & C 2 & 1993 & 21 & 2004 & août 2016 & Erbil \\
\hline Beste & 43 & C 2 & 1990 & 18 & & août 2016 & Souleymanieh \\
\hline Shilan & 42 & C 2 & 1992 & 17 & & mars 2017 & Kandil \\
\hline Giçek & 42 & G 2 & 1992 & 16 & & mars 2017 & Kandil \\
\hline Hayet & 41 & C 2 & 1991 & 14 & & août 2016 & Kandil \\
\hline Vickie & 40 & C 2 & 1990 & 13 & & août 2016 & Kandil \\
\hline Nujin & 40 & G 2 & 1992 & 12 & 2007 & août 2016 & Erbil \\
\hline Irène & 40 & G 2 & - & - & & août 2016 & Kandil \\
\hline Rewsen & 40 & C 2 & 1992 & 14 & & mars 2017 & Kandil \\
\hline Sofiana & 38 & C 2 & 1993 & 15 & & août 2016 & Kandil \\
\hline Gentiane & 38 & C 3 & 2001 & 23 & & août 2016 & Kandil \\
\hline Yekta & 37 & C 2 & 1993 & 17 & & mars 2017 & Kandil \\
\hline $\begin{array}{l}\text { Medya- } \\
\text { Térésa }\end{array}$ & 37 & C 2 & 1992 & 13 & & août 2016 & Kandil \\
\hline Alexia & 34 & C 3 & 2003 & 21 & & août 2016 & Kandil \\
\hline Rojen & 34 & C 3 & $\begin{array}{l}\text { Début des } \\
2000 \text { 's }\end{array}$ & 17 & & mars 2017 & Kandil \\
\hline Victoria & 34 & C 3 & 2004 & 22 & & août 2016 & Kandil \\
\hline Olga & 33 & C 3 & 1999 & 16 & & août 2016 & Kandil \\
\hline Berivan & 32 & C 3 & 2013 & 28 & & mars 2017 & Kandil \\
\hline Lilas & 31 & C 3 & 2005 & 20 & & août 2016 & Kandil \\
\hline Berbiheyv & - & C 3 & 2005 & - & & 2017 & - \\
\hline Coline & 30 & C 3 & 2009 & 23 & & 2016 & Souleymanieh \\
\hline Viyan & 27 & C 3 & 2009 & 19 & & mars 2017 & Kandil \\
\hline Idra & 23 & C 3 & 2013 & 19 & & août 2016 & Kandil \\
\hline Primevère & 22 & C 3 & 2013 & 19 & & 2016 & Souleymanieh \\
\hline
\end{tabular}

35 Les archives électroniques des deux publications mensuelles sont disponibles sur http://www.serxwebun.org/index.php?sys=arsiv. 